Research Article

\title{
The Centrosymmetric Matrices of Constrained Inverse Eigenproblem and Optimal Approximation Problem
}

\author{
Jinrong Shen $(\mathbb{D}$, Wei Liu, Baiyu Wang, and Xiangyang Peng \\ College of Computer Engineering and Applied Mathematics, Changsha University, Changsha 410003, China \\ Correspondence should be addressed to Jinrong Shen; ccsusjr@163.com
}

Received 20 December 2019; Revised 31 January 2020; Accepted 11 February 2020; Published 10 March 2020

Academic Editor: Ruben Specogna

Copyright ( 2020 Jinrong Shen et al. This is an open access article distributed under the Creative Commons Attribution License, which permits unrestricted use, distribution, and reproduction in any medium, provided the original work is properly cited.

In this paper, a kind of constrained inverse eigenproblem and optimal approximation problem for centrosymmetric matrices are considered. Necessary and sufficient conditions of the solvability for the constrained inverse eigenproblem of centrosymmetric matrices in real number field are derived. A general representation of the solution is presented for a solvable case. The explicit expression of the optimal approximation problem is provided. Finally, a numerical example is given to illustrate the effectiveness of the method.

\section{Introduction}

Inverse eigenproblems arise in a remarkable variety of applications, including control theory $[1,2]$, vibration theory $[3,4]$, structural design [5], molecular spectroscopy [6], in developing numerical methods, and the ordinary and partial differential equation solving $[7,8]$. Centrosymmetric matrices are applied in information theory, linear system theory, and numerical analysis theory [9]. The unconstrained centrosymmetric matrices' problems have been discussed [9-14], a class of unconstrained matrices' inverse eigenproblems has been obtained [15-18], and the constrained inverse eigenproblems have been discussed [19-22], but only when the eigenvalues are real or imaginary numbers. For general real matrices, the eigenvalues are not necessarily real or imaginary numbers, so when the eigenvalue is complex, it is difficult to find the constraint solution. In this paper, we will use the real Schur decomposition theorem and the similar decomposition theorem and introduce a new norm to get the corresponding expression of the best approximation solution.

Throughout the paper, we denote the set of real $n \times m$ matrices, real $n \times n$ orthogonal matrices, $n \times n$ centrosymmetric matrices, and real numbers, respectively, by $R^{n \times m}$, $\mathrm{OR}^{n \times n}, \mathrm{CSR}^{n \times n}$, and $R . A^{T}, A^{+}, \operatorname{rank}(A)$, and $\|A\|_{F}$ denote the transpose, the Moore-Penrose generalized inverse, the rank, and the Frobenius norm of a matrix $A$, respectively. $I$ is the identity matrix. $\lambda(A)$ denotes the set of eigenvalues of the matrix $A . \lambda(A) / \lambda(B)$ denotes the set of the difference of $\lambda(A)$ and $\lambda(B) . D_{r}$ is a closed disc with radius $r$ and center origin, $[A]_{D_{r}}$ denotes the square matrix $A$ with all of its eigenvalues located in the closed disc $D_{r}$, and $R_{D_{r}}^{n \times n}$ denotes the set of $n \times n$ matrices with their eigenvalues located in the disc $D_{r}$. The notation $A_{11} \oplus A_{22} \oplus \cdots \oplus A_{k k}$ denotes the direct sum of the matrices $A_{11}, A_{22}, \ldots, A_{k k}$, where $A_{j j} \in R^{n_{j} \times n_{j}}$. Let $e_{i}$ be the $i$ th standard unit vector, and the matrix $S_{n}=\left(e_{n}, e_{n-1}, \ldots, e_{1}\right) .[x]$ represents the largest integer less than or equal to $x$. The following definition is given in [23].

Definition 1. $A=\left(a_{i j}\right) \in R^{n \times n}$ is called centrosymmetric matrix if $a_{i j}=a_{n+1-i, n+1-j}, i, j=1,2, \ldots, n$.

Clearly, each eigenvalue of matrix $A \in \operatorname{CSR}^{n \times n}$ is either a real number or a complex number, if $A$ has complex eigenvalues, and they must occur in complex conjugate pairs, note that $\lambda_{j}=\widetilde{a}_{j}+i \widetilde{b}_{j}, \bar{\lambda}_{j}=\widetilde{a}_{j}-i \widetilde{b}_{j}\left(\vec{b}_{j} \neq 0, j=1,2, \ldots, k\right)$ are eigenvalues of $A, x_{j}=\xi_{j}+\eta_{j} i$ and $\bar{x}_{j}=\xi_{j}-\eta_{j} i(j=1,2, \ldots, k)$ are eigenvectors associated with $\lambda_{j}$ and $\bar{\lambda}_{j}$, respectively; also, we have

$$
A\left[\xi_{j} \eta_{j}\right]=\left[\xi_{j} \eta_{j}\right]\left[\begin{array}{cc}
\tilde{a}_{j} & \tilde{b}_{j} \\
-\tilde{b}_{j} & \tilde{a}_{j}
\end{array}\right], \quad j=1,2, \ldots, k,
$$

where let 


$$
\begin{aligned}
X_{j} & =\left[\xi_{j}, \eta_{j}\right], \\
\Lambda_{j} & =\left[\begin{array}{cc}
\widetilde{a}_{j} & \widetilde{b}_{j} \\
-\widetilde{b}_{j} & \tilde{a}_{j}
\end{array}\right] .
\end{aligned}
$$

If $A$ has real eigenvalues, then $\lambda_{j} \in R(j=k+1, \ldots, l)$ are eigenvalues of $A$ and $x_{j} \in R^{n}(j=k+1, \ldots, l)$ are eigenvectors associated with $\lambda_{j}$; also, we have

$$
A x_{j}=\lambda_{j} x_{j}, \quad j=k+1, \ldots, l,
$$

where let

$$
\begin{aligned}
& X_{j}=x_{j}, \\
& \Lambda_{j}=\lambda_{j},
\end{aligned}
$$

Then, $2 k+(l-k)=n$.

Remark 1 . Here, $r$-multiple eigenvalues are counted $r$-times and their eigenvectors may be linearly dependent.

Definition 2. Let $A \in R^{n \times n}$, given $Y \in R^{n \times n}$, and $\operatorname{rank}(Y)=n$. Then, we define a new norm called the $Y$ norm of matrix $A$ as

$$
\|A\|_{Y}=\left\|Y^{-1} A Y\right\|_{F} .
$$

In contrast to the definition of matrix norm (see [24], Definition 5.1.1), it is easy to show that $\|\cdot\|_{Y}$ is a kind of matrix norm.

Now, we can present the optimal approximation of constrained inverse eigenproblem of centrosymmetric matrices as follows:

Constrained Inverse Eigenproblem. Given a real number $r>0$. Let $X=\left(X_{1}, X_{2}, \ldots, X_{l}\right) \in R^{n \times m}, \Lambda=\operatorname{diag}\left(\Lambda_{1}\right.$, $\left.\Lambda_{2}, \ldots, \Lambda_{l}\right) \in R^{m \times m}, l \leq m \leq n$, where $X_{j}$ and $\Lambda_{j}$ satisfy equation (2) or equation (4). $D_{r}=\{Z|| Z \mid \leq r, Z \in C\}$ is a given closed disc. Find matrix $A$ such that the set

$$
S=\left\{A \in \operatorname{CSR}^{n \times n} \mid A X=X \Lambda\right\} .
$$

is nonempty, and find the subset $S_{D_{r}} \subset S$ such that the remaining eigenvalues of any matrix in $S$ are located in the disc $D_{r}$.

Optimal Approximation Problem. Given $A^{*} \in R^{n \times n}$, $\|\cdot\|_{Y}$ is the norm which has been defined as equation (5), $Y$ is an invertible matrix concerning with $A^{*}$ and $A \in S_{D_{r}}$; find a matrix $\widehat{A} \in S_{D_{r}}$, such that

$$
\left\|A^{*}-\widehat{A}\right\|_{Y}=\min _{\forall A \in S_{D_{r}}}\left\|A^{*}-A\right\|_{Y} \text {, }
$$

where $S_{D_{r}}$ is the solution set of the Constrained Inverse Eigenproblem.

This paper is organized as follows. In Section 2, we provide the solvability conditions of the Constrained Inverse Eigenproblem and its general solution in that case. In Section 3, we get the expression of the solution for Optimal Approximation Problem. In Section 4, we give an algorithm of Constrained Inverse Eigenproblem and Optimal
Approximation Problem and give a numerical example of Optimal Approximation Problem.

\section{The Solvability Conditions and General Solution of Constrained Inverse Eigenproblem}

Firstly, let $k=[n / 2]$ and characterize the set of all centrosymmetric matrices as follows.

When $n=2 k$, let

$$
D=\frac{1}{\sqrt{2}}\left[\begin{array}{cc}
I_{k} & I_{k} \\
S_{k} & -S_{k}
\end{array}\right]
$$

When $n=2 k+1$, let

$$
D=\frac{1}{\sqrt{2}}\left[\begin{array}{ccc}
I_{k} & 0 & I_{k} \\
0 & \sqrt{2} & 0 \\
S_{k} & 0 & -S_{k}
\end{array}\right] .
$$

Clearly, $D$ is an orthogonal matrix for all of the $n$.

Secondly, given $X \in R^{n \times m}$, denote

$$
D^{T} X=\left[\begin{array}{c}
X_{D_{1}} \\
X_{D_{2}}
\end{array}\right], \quad X_{D_{1}} \in R^{(n-k) \times m}, X_{D_{2}} \in R^{k \times m} .
$$

Decomposing the matrices $X, X_{D_{1}}$, and $X_{D_{2}}$ by the SVD, we have

$$
X=\widetilde{U}\left[\begin{array}{ll}
\Delta & 0 \\
0 & 0
\end{array}\right] \widetilde{V}^{T}=\widetilde{U}_{1} \Delta \widetilde{V}_{1}^{T},
$$

where $\widetilde{U}=\left[\widetilde{U}_{1}, \widetilde{U}_{2}\right] \in \mathrm{OR}^{n \times n}, \widetilde{V}=\left[\widetilde{V}_{1}, \widetilde{V}_{2}\right] \in \mathrm{OR}^{m \times m}, r_{0}=$ $\operatorname{rank}(X)$, and

$$
\begin{aligned}
\Delta & =\operatorname{diag}\left(\delta_{1}, \delta_{2}, \ldots, \delta_{r_{0}}\right), \quad \delta_{i}>0,1 \leq i \leq r_{0}, \\
X_{D_{1}} & =U\left[\begin{array}{ll}
\Gamma & 0 \\
0 & 0
\end{array}\right] V^{T}=U_{1} \Gamma V_{1}^{T},
\end{aligned}
$$

where $\quad U=\left[U_{1}, U_{2}\right] \in \mathrm{OR}^{(n-k) \times(n-k)}, V=\left[V_{1}, V_{2}\right] \epsilon$ $\mathrm{OR}^{m \times m}, r_{1}=\operatorname{rank}\left(X_{D_{1}}\right), \quad$ and $\quad \Gamma=\operatorname{diag}\left(\gamma_{1}, \gamma_{2}, \ldots, \gamma_{r_{1}}\right)$, $\left(\gamma_{i}>0,1 \leq i \leq r_{1}\right)$.

$$
X_{D_{2}}=P\left[\begin{array}{ll}
\Sigma & 0 \\
0 & 0
\end{array}\right] Q^{T}=P_{1} \Sigma Q_{1}^{T},
$$

where $P=\left[P_{1}, P_{2}\right] \in \mathrm{OR}^{k \times k}, Q=\left[Q_{1}, Q_{2}\right] \in \mathrm{OR}^{m \times m}, r_{2}=$ $\operatorname{rank}\left(X_{D_{2}}\right)$, and $\Sigma=\operatorname{diag}\left(\sigma_{1}, \sigma_{2}, \ldots, \sigma_{r_{2}}\right),\left(\sigma_{i}>0,1 \leq i \leq r_{2}\right)$.

Lemma 1 (see [9], Lemma 2). A $\in C^{n \times n}$ if and only if $A$ can be expressed as

$$
A=D\left[\begin{array}{ll}
B & 0 \\
0 & C
\end{array}\right] D^{T}, \quad B \in R^{(n-k) \times(n-k)}, C \in R^{k \times k},
$$

where, if $n=2 k$, then $D$ is the form of equation (8), and if $n=2 k+1$, then $D$ is the form of (9). 
Lemma 2 (see [15], Theorems 7.2 and 7.3). Given $X \in R^{n \times m}, \Lambda \in R^{m \times m}$, and $X$ decomposed as equation (11), let

$$
\widetilde{U}^{T} A \widetilde{U}=\left[\begin{array}{ll}
A_{11} & A_{12} \\
A_{21} & A_{22}
\end{array}\right],
$$

where $A_{i j}=\widetilde{U}_{i}^{T} A \widetilde{U}_{j}(i, j=1,2)$. Equation $A X=X \Lambda$ is consistent if and only if

$$
X \Lambda \widetilde{V}_{2}=0 .
$$

If the condition is satisfied, the general solution can be expressed as

$$
A=X \Lambda X^{+}+G \widetilde{U}_{2}^{T}
$$

and $G=\widetilde{U}\left[\begin{array}{l}A_{12} \\ A_{22}\end{array}\right] \in R^{n \times\left(n-r_{0}\right)}$ is arbitrary.

Lemma 3. Given $X \in R^{n \times m}$ and $\Lambda \in R^{m \times m}$. Equation $A X=$ $X \Lambda$ is consistent with $A \in C S R^{n \times n}$ if and only if

$$
\begin{aligned}
& X_{D_{1}} \Lambda V_{2}=0, \\
& X_{D_{2}} \Lambda Q_{2}=0,
\end{aligned}
$$

where $X_{D_{1}}, X_{D_{2}}, V_{2}$, and $Q_{2}$ is the same as equations (10)-(14). Let

$$
\begin{aligned}
U^{T} B U & =\left[\begin{array}{ll}
B_{11} & B_{12} \\
B_{21} & B_{22}
\end{array}\right], \\
P^{T} C P & =\left[\begin{array}{ll}
C_{11} & C_{12} \\
C_{21} & C_{22}
\end{array}\right],
\end{aligned}
$$

where $B_{i j}=U_{i}^{T} B U_{j}$ and $C_{i j}=P_{i}^{T} C P_{j}(i, j=1,2)$, and the set of the general solution can be expressed as

$$
S=\left\{A \mid A=D\left[\begin{array}{cc}
X_{D_{1}} \Lambda X_{D_{1}}^{+}+G_{1} U_{2}^{T} & 0 \\
0 & X_{D_{2}} \Lambda X_{D_{2}}^{+}+G_{2} P_{2}^{T}
\end{array}\right] D^{T}\right\},
$$

where $G_{1}=U\left[\begin{array}{l}B_{12} \\ B_{22}\end{array}\right] \in R^{(n-k) \times\left(n-k-r_{1}\right)}$ and $G_{2}=P\left[\begin{array}{l}C_{12} \\ C_{22}\end{array}\right] \epsilon$ $R^{k \times\left(k-r_{2}\right)}$ are arbitrary.

Proof. If $S$ is nonempty, by Lemma 1, we have

$$
A=D\left[\begin{array}{ll}
B & 0 \\
0 & C
\end{array}\right] D^{T}, \quad B \in R^{(n-k) \times(n-k)}, C \in R^{k \times k} .
$$

Using equation (10), equation $A X=X \Lambda$ is equivalent to

$$
\begin{aligned}
& B X_{D_{1}}=X_{D_{1}} \Lambda, \\
& C X_{D_{2}}=X_{D_{2}} \Lambda .
\end{aligned}
$$

It follows from Lemma 2 , and we can obtain $A X=X \Lambda$ is solvable if and only if equation (19) holds, and the solution set can be expressed as equation (21) easily.

Remark 2. Equation (21) implies that $\lambda(A) / \lambda(\Lambda) \in S_{D_{r}}$ if and only if arbitrary matrices $\lambda\left(B_{22}\right) \in S_{D_{r}}$ and $\lambda\left(C_{22}\right) \in S_{D_{r}}$. From Lemmas 1-3, we have the following theorem.
Theorem 1. Constrained Inverse Eigenproblem is solvable if and only if

$$
\begin{aligned}
& X_{D_{1}} \Lambda V_{2}=0, \\
& X_{D_{2}} \Lambda Q_{2}=0 .
\end{aligned}
$$

The general solution can be expressed as

$$
A=D\left[\begin{array}{cc}
X_{D_{1}} \Lambda X_{D_{1}}^{+}+U\left[\begin{array}{c}
B_{12} \\
{\left[B_{22}\right]_{D_{r}}}
\end{array}\right] & 0 \\
0 & X_{D_{2}} \Lambda X_{D_{2}}^{+}+P\left[\begin{array}{c}
C_{12} \\
{\left[C_{22}\right]_{D_{r}}}
\end{array}\right]
\end{array}\right] D^{T},
$$

where $\quad B_{12} \in R^{r_{1} \times\left(n-k-r_{1}\right)}, \quad C_{12} \in R^{r_{2} \times\left(k-r_{2}\right)}$, $\left[B_{22}\right]_{D_{r}} \in R_{D_{r}}^{\left(n-k-r_{1}\right) \times\left(n-k-r_{1}\right)}$, and $\left[C_{22}\right]_{D_{r}} \in R_{D_{r}}^{\left(k-r_{2}\right) \times\left(k-r_{2}\right)}$ are arbitrary matrices.

\section{The Solution of Optimal Approximation Problem}

Lemma 5. Given a matrix

$$
\begin{aligned}
\widetilde{B}_{j j} & =\left[\begin{array}{cc}
c_{j} & d_{1 j} \\
-d_{2 j} & c_{j}
\end{array}\right], \\
d_{1 j} d_{2 j} & >0, \\
\left|\lambda\left(\widetilde{B}_{j j}\right)\right|^{2} & =c_{j}^{2}+d_{1 j} d_{2 j} .
\end{aligned}
$$

By similarity invariant, we can derive an invertible matrix $Y_{\widetilde{B}_{j j}}$ and $\left\|Y_{\widetilde{B}_{j j}}\right\|_{F}=1$ such that

$$
\widetilde{B}_{j j}=Y_{\widetilde{B}_{j j}} B_{j j} Y_{\widetilde{B}_{j j}}^{-1},
$$

where $B_{j j}=\left[\begin{array}{cc}c_{j} & d_{j} \\ -d_{j} & c_{j}\end{array}\right]$ and $\left|\lambda\left(B_{j j}\right)\right|^{2}=c_{j}^{2}+d_{j}^{2}=\left|\lambda\left(\widetilde{B}_{j j}\right)\right|^{2}$. Lemma Given $B_{j j}=\left[\begin{array}{cc}c_{j} & d_{j} \\ -d_{j} & c_{j}\end{array}\right]$ and $\left|\lambda\left(B_{j j}\right)\right|=$ $\sqrt{c_{j}^{2}+d_{j}^{2}}=s_{j}>r>0$, then, there exists a unique matrix:

$$
A_{j j}=\left[\begin{array}{cc}
a_{j} & b_{j} \\
-b_{j} & a_{j}
\end{array}\right], \sqrt{a_{j}^{2}+b_{j}^{2}}=r,
$$

such that

$$
\begin{aligned}
\left\|A_{j j}-B_{j j}\right\|_{F} & =\min , \\
A_{j j} & =\frac{r}{\left|\lambda\left(B_{j j}\right)\right|} B_{j j} .
\end{aligned}
$$

Proof. From

$$
\left\|A_{j j}-B_{j j}\right\|_{F}^{2}=\min ,
$$

we may obtain 


$$
\left(a_{j}-c_{j}\right)^{2}+\left(b_{j}-d_{j}\right)^{2}=\min .
$$

Let $\left(d_{j} / c_{j}\right)=\tan \theta_{j},\left(b_{j} / a_{j}\right)=\tan \theta, 0 \leq \theta, \theta_{j} \leq \pi, s_{j}>r$, then equation (32) is equivalent to

$$
\begin{aligned}
\min f(\theta, r) & =\min \left(r \cos \theta-s_{j} \cos \theta_{j}\right)^{2}+\left(r \sin \theta-s_{j} \sin \theta_{j}\right)^{2} \\
& =\min \left(r^{2}+s_{j}^{2}-2 r s_{j} \cos \left(\theta-\theta_{j}\right)\right) .
\end{aligned}
$$

It is easy to see that $f(\theta, r)=\min$ if and only if $\theta=\theta_{j}$ and $r=s_{j}$.

Note $\left[B_{j j}\right]_{D_{r}}=A_{j j}$, from equation (33), we may show that equation (29) implies that

$$
\left[B_{j j}\right]_{D_{r}}=A_{j j}=\frac{r}{s_{j}} B_{j j}=\frac{r}{\left|\lambda\left(B_{j j}\right)\right|} B_{j j} .
$$

There, we note $\left[B_{j j}\right]_{D_{r}}=A_{j j}$ just for the sake of writing.

To show Theorem 2, we first introduce some notations as follows:

$$
D^{T} A^{*} D=\left[\begin{array}{cc}
A_{11}^{*} & A_{12}^{*} \\
A_{21}^{*} & A_{22}^{*}
\end{array}\right],
$$

where $\quad A_{11}^{*} \in R^{(n-k) \times(n-k)}, \quad A_{12}^{*} \in R^{(n-k) \times k}, A_{21}^{*} \in R^{k \times(n-k)}$, and $A_{22}^{*} \in R^{k \times k}$.

Lemma 7 (see [16], Theorem 7.6). If $H \in R^{n \times n}$, then there exists an orthogonal matrix $\widetilde{Q} \in O R^{n \times n}$, such that $\widetilde{Q}^{T} H \widetilde{Q}$ is upper quasi-triangular, that is, $\widetilde{Q}^{T} H \widetilde{Q}$ is block upper-triangular where each diagonal block is either a $1 \times 1$ matrix or a $2 \times 2$ matrix having complex conjugate eigenvalues.

Lemma 8. Given matrix $A^{*} \in R^{n \times n}, D^{T} A^{*} D$ is decomposed as equation (35), the orthogonal matrices $U$ and $P$ are given in equations (13) and (14), respectively; then, there exist orthogonal matrices $Q_{1}^{*}$ and $Q_{2}^{*}$ and inverse matrices $Y_{1}$ and $Y_{2}$, such that $U_{2}^{T} A_{11}^{*} U_{2}=Q_{1}^{*^{T}} Y_{1}\left(\begin{array}{cccccc}\bar{\Lambda}_{11} & * & \cdots & \cdots & \cdots & * \\ 0 & \ddots & \ddots & & & \vdots \\ \vdots & \ddots & \bar{\Lambda}_{s s} & \ddots & & \vdots \\ \vdots & & \ddots & \lambda_{s+1} & \ddots & \vdots \\ \vdots & & & \ddots & \ddots & * \\ 0 & \cdots & \cdots & \cdots & 0 & \lambda_{n-k-r_{1}-2 s}\end{array}\right) Y_{1}^{-1} Q_{1}^{*}$

where $\bar{\Lambda}_{j j}=\left(\begin{array}{cc}l_{j} & f_{j} \\ -f_{j} & l_{j}\end{array}\right), j=1,2, \ldots, s$.

$$
P_{2}^{T} A_{22}^{*} P_{2}=Q_{2}^{*^{T}} Y_{2}\left(\begin{array}{cccccc}
\bar{\Gamma}_{11} & * & \cdots & \cdots & \cdots & * \\
0 & \ddots & \ddots & & & \vdots \\
\vdots & \ddots & \bar{\Gamma}_{t t} & \ddots & & \vdots \\
\vdots & & \ddots & \mu_{t+1} & \ddots & \vdots \\
\vdots & & & \ddots & \ddots & * \\
0 & \cdots & \cdots & \cdots & 0 & \mu_{k-r_{2}-2 t}
\end{array}\right) Y_{2}^{-1} Q_{2}^{*}
$$

where $\bar{\Gamma}_{j j}=\left(\begin{array}{cc}g_{j} & h_{j} \\ -h_{j} & g_{j}\end{array}\right), j=1,2, \ldots, t$.
Proof. For Lemma 7, there exist orthogonal matrices $Q_{1}^{*} \in \mathrm{OR}^{\left(n-k-r_{1}\right) \times\left(n-k-r_{1}\right)}$ and $Q_{2}^{*} \in \mathrm{OR}^{\left(k-r_{2}\right) \times\left(k-r_{2}\right)}$ such that

$$
\begin{aligned}
& U_{2}^{T} A_{11}^{*} U_{2}=Q_{1}^{*^{T}}\left(\begin{array}{cccccc}
\Lambda_{11} & * & \cdots & \cdots & \cdots & * \\
0 & \ddots & \ddots & & & \vdots \\
\vdots & \ddots & \Lambda_{s s} & \ddots & & \vdots \\
\vdots & & \ddots & \lambda_{s+1} & \ddots & \vdots \\
\vdots & & & \ddots & \ddots & * \\
0 & \cdots & \cdots & \cdots & 0 & \lambda_{n-k-r_{1}-2 s}
\end{array}\right) Q_{1}^{*}, \\
& P_{2}^{T} A_{22}^{*} P_{2}=Q_{2}^{*^{T}}\left(\begin{array}{cccccc}
\Gamma_{11} & * & \cdots & \cdots & \cdots & * \\
0 & \ddots & \ddots & & & \vdots \\
\vdots & \ddots & \Gamma_{t t} & \ddots & & \vdots \\
\vdots & & \ddots & \mu_{t+1} & \ddots & \vdots \\
\vdots & & & \ddots & \ddots & * \\
0 & \cdots & \cdots & \cdots & 0 & \mu_{k-r_{2}-2 t}
\end{array}\right) Q_{2}^{*},
\end{aligned}
$$

where

$$
\begin{aligned}
& \Lambda_{j j}=\left(\begin{array}{cc}
l_{j} & f_{1 j} \\
-f_{2 j} & l_{j}
\end{array}\right), \quad \lambda_{j} \in R, j=s+1, \ldots, n-k-r_{1}-2 s, \\
& \Gamma_{j j}=\left(\begin{array}{cc}
g_{j} & h_{1 j} \\
-h_{2 j} & g_{j}
\end{array}\right), \quad \mu_{j} \in R, j=t+1, \ldots, k-r_{2}-2 t .
\end{aligned}
$$

For Lemma 5, there exist inverse matrices $Y_{1 j}$ and $Y_{2 j}$, satisfying

$$
\begin{aligned}
Y_{1 j}^{-1} \Lambda_{j j} Y_{1 j} & =\left(\begin{array}{cc}
l_{j} & f_{j} \\
-f_{j} & l_{j}
\end{array}\right)=\bar{\Lambda}_{j j}, \\
\left\|Y_{1 j}\right\|_{F} & =1, \quad j=1,2, \ldots, s, \\
Y_{2 j}^{-1} \Gamma_{j j} Y_{2 j} & =\left(\begin{array}{cc}
g_{j} & h_{j} \\
-h_{j} & g_{j}
\end{array}\right)=\bar{\Gamma}_{j j}, \\
\left\|Y_{2 j}\right\|_{F} & =1, \quad j=1,2, \ldots, t .
\end{aligned}
$$

Let $\quad Y_{1}=Y_{11} \oplus \cdots \oplus Y_{1 s} \oplus I_{n-k-r_{1}-2 s} \quad$ and $Y_{2}=Y_{21} \oplus \cdots \oplus Y_{2 t} \oplus I_{k-r_{2}-2 t}, \quad I_{n-k-r_{1}-2 s}$ and $I_{k-r_{2}-2 t}$ are identity matrices. Then, it is easy to see that equations (36) and (37) exist.

Theorem 2. Given $A^{*} \in R^{n \times n}$, suppose given matrices $X$ and $\Lambda$, real number $r>0$ make Constrained Inverse Eigenproblem solvable, and the solution expression is the same as equation (25). Let 


$$
Y=D\left(\begin{array}{ll}
U & 0 \\
0 & P
\end{array}\right)\left(\begin{array}{cccc}
I_{r_{1}} & 0 & 0 & 0 \\
0 & Q_{1}^{* T} Y_{1} & 0 & 0 \\
0 & 0 & I_{r_{2}} & 0 \\
0 & 0 & 0 & Q_{2}^{*^{T}} Y_{2}
\end{array}\right) .
$$

Then, Optimal Approximation Problem has a unique solution which can be expressed as

$$
\widehat{A}=D\left(\begin{array}{ll}
\widehat{B} & 0 \\
0 & \widehat{C}
\end{array}\right) D^{T},
$$

where

$$
\begin{aligned}
& \widehat{B}=U\left(\begin{array}{cc}
\Sigma_{1} V_{1}^{T} \Lambda V_{1} \Sigma_{1}^{-1} & U_{1}^{T} A_{11}^{*} U_{2} \\
0 & {\left[B_{22}\right]_{D_{r}}}
\end{array}\right) U^{T}, \\
& \widehat{C}=P\left(\begin{array}{cc}
\Sigma_{2} Q_{1}^{T} \Lambda Q_{1} \Sigma_{2}^{-1} & P_{1}^{T} A_{22}^{*} P_{2} \\
0 & {\left[C_{22}\right]_{D_{r}}}
\end{array}\right) P^{T} .
\end{aligned}
$$

Proof. Let $A \in S_{D_{r}}$, by Definition 2, we have

$$
\left\|A-A^{*}\right\|_{Y}^{2}=\left\|Y^{-1} A Y-Y^{-1} A^{*} Y\right\|_{F}^{2}=\min _{\forall A \in S_{D_{r}}} .
$$

The expression of $\mathrm{A}$ is the same as equation (25) and $D^{T} A^{*} D$ is decomposed as equation (35); then, equation (44) is equivalent to

$$
\begin{aligned}
& \left\|\Sigma_{1} V_{1}^{T} \Lambda V_{1} \Sigma_{1}^{-1}-U_{1}^{T} A_{11}^{*} U_{1}\right\|^{2}+\left\|B_{12} Q_{1}^{*^{T}} Y_{1}-U_{1}^{T} A_{11}^{*} U_{2} Q_{1}^{*^{T}} Y_{1}\right\|^{2}+\left\|Y_{1}^{-1} Q_{1}^{*}\left[B_{22}\right]_{D_{r}} Q_{1}^{*^{T}} Y_{1}-Y_{1}^{-1} Q_{1}^{*} U_{2}^{T} A_{11}^{*} U_{2} Q_{1}^{*} Y_{1}\right\|^{2} \\
& \quad+\left\|\Sigma_{2} Q_{1}^{T} \Lambda Q_{1} \Sigma_{2}^{-1}-P_{1}^{T} A_{22}^{*} P_{1}\right\|^{2}+\left\|C_{12} Q_{12}^{*^{T}} Y_{2}-P_{1}^{T} A_{22}^{*} P_{2} Q_{2}^{*^{T}} Y_{2}\right\|^{2}+\left\|Y_{2}^{-1} Q_{2}^{*}\left[C_{22}\right]_{D_{r}} Q_{2}^{*^{T}} Y_{2}-Y_{2}^{-1} Q_{2}^{*} P_{2}^{T} A_{22}^{*} P_{2} Q_{2}^{*^{T}} Y_{2}\right\|^{2} \\
& =\min .
\end{aligned}
$$

Obviously, we may derive that equation (44) has the solution $\widehat{A}$ if and only if

$$
\begin{gathered}
\left\|Y_{1}^{-1} Q_{1}^{*} U_{2}^{T} A_{11}^{*} U_{2} Q_{1}^{*^{T}} Y_{1}-Y_{1}^{-1} Q_{1}^{*}\left[B_{22}\right]_{D_{r}} Q_{1}^{*^{T}} Y\right\|_{F}^{2}=\min \\
\left\|Y_{2}^{-1} Q_{2}^{*} P_{2}^{T} A_{22}^{*} P_{2} Q_{2}^{*^{T}} Y_{2}-Y_{2}^{-1} Q_{2}^{*}\left[C_{22}\right]_{D_{r}} Q_{2}^{*} Y_{2}\right\|_{F}^{2}=\min \\
\left\|U_{1}^{T} A_{11}^{*} U_{2} Q_{1}^{*^{T}} Y_{1}-B_{12} Q_{1}^{*^{T}} Y_{1}\right\|_{F}=\min
\end{gathered}
$$

$$
\left\|P_{1}^{T} A_{22}^{*} P_{2} Q_{2}^{*^{T}} Y_{2}-C_{12} Q_{2}^{*^{T}} Y_{2}\right\|_{F}=\min .
$$

From Lemmas 5 and 6, equations (36) and (37), we may see that equations (46) and (47) imply that

$$
\left[B_{22}\right]_{D_{r}}=Q_{1}^{*^{T}} Y_{1}\left(\begin{array}{cccccc}
{\left[\bar{\Lambda}_{11}\right]_{D_{r}}} & * & \ldots & \ldots & \ldots & * \\
0 & \ddots & \ddots & & & \vdots \\
\vdots & \ddots & {\left[\bar{\Lambda}_{s s}\right]_{D_{r}}} & \ddots & & \vdots \\
\vdots & & \ddots & {\left[\bar{\lambda}_{s+1}\right]_{D_{r}}} & \ddots & \vdots \\
\vdots & & & \ddots & \ddots & * \\
0 & \ldots & \ldots & \ldots & 0 & {\left[\bar{\lambda}_{n-k-r_{1}-2 s}\right]_{D_{r}}}
\end{array}\right) Y_{1}^{-1} Q_{1}^{*}
$$


Step 1. Input $X, \Lambda, A^{*}, r$;

Step 2. Decompose $D^{T} X$ as equation (10);

Step 3. Calculate the singular value decomposition $X_{D_{1}}, X_{D_{2}}$ as equations (13) and (14), verify that equation (24) is true and $A$ can be expressed as equation (25);

Step 4. Decompose $D^{T} A^{*} D$ as equation (35);

Step 5. Decompose $U_{2}^{T} A_{11}^{*} U_{2}, P_{2}^{T} A_{22}^{*} P_{2}$ as equations (36), (37), respectively;

Step 6. Calculate $\left[B_{22}\right]_{D_{r}},\left[C_{22}\right]_{D_{r}}$ as equations (50) and (52), respectively;

Step 7. Calculate $\widehat{A}$ by Theorem 2 .

Algorithm 1: The solution of (7).

where

$$
\begin{aligned}
& {\left[\bar{\Lambda}_{j j}\right]_{D_{r}}= \begin{cases}\frac{r}{\lambda\left(\bar{\Lambda}_{j j}\right)} \bar{\Lambda}_{j j}, & \left|\lambda\left(\bar{\Lambda}_{j j}\right)\right|>r \\
\bar{\Lambda}_{j j}, & \left|\lambda\left(\bar{\Lambda}_{j j}\right)\right| \leq r\end{cases} } \\
& {\left[\bar{\lambda}_{j j}\right]_{D_{r}}= \begin{cases}\operatorname{sign}\left(\bar{\lambda}_{i}\right) r, & \left|\lambda_{i}\right|>r, \\
\lambda_{i}, & \left|\lambda_{i}\right| \leq r,\end{cases} } \\
& j=1,2, \ldots, s, i=s+1, \ldots, n-k-r_{1}-2 s . \\
& {\left[C_{22}\right]_{D_{r}}=Q_{2}^{*^{T}} Y_{2}\left(\begin{array}{cccccc}
{\left[\bar{\Gamma}_{11}\right]_{D_{r}}} & * & \cdots & \cdots & \cdots & * \\
0 & \ddots & \ddots & & & \vdots \\
\vdots & \ddots & {\left[\bar{\Gamma}_{t t}\right]_{D_{r}}} & \ddots & & \vdots \\
\vdots & & \ddots & {\left[\bar{\mu}_{t+1}\right]_{D_{r}}} & \ddots & \vdots \\
\vdots & & & \ddots & \ddots & * \\
0 & \ldots & \cdots & \cdots & 0 & {\left[\bar{\mu}_{k-r_{2}-2 t}\right]_{D_{r}}}
\end{array}\right) Y_{2}^{-1} Q_{2}^{*},}
\end{aligned}
$$

where

$$
\begin{aligned}
{\left[\bar{\Gamma}_{j j}\right]_{D_{r}}= \begin{cases}\frac{r}{\lambda\left(\bar{\Gamma}_{j j}\right)} \bar{\Gamma}_{j j}, & \left|\lambda\left(\bar{\Gamma}_{j j}\right)\right|>r, \\
\bar{\Gamma}_{j j}, & \left|\lambda\left(\bar{\Gamma}_{j j}\right)\right| \leq r,\end{cases} } \\
{\left[\bar{\mu}_{j j}\right]_{D_{r}}= \begin{cases}\operatorname{sign}\left(\bar{\mu}_{i}\right) r, & \left|\mu_{i}\right|>r, \\
\bar{\mu}_{i}, & \left|\mu_{i}\right| \leq r,\end{cases} } \\
j=1, \ldots, t, i=t+1, \ldots, k-r_{2}-2 t .
\end{aligned}
$$

By equations (48) and (49), we have

$$
\begin{aligned}
& B_{12}=U_{1}^{T} A_{11}^{*} U_{2}, \\
& C_{12}=P_{1}^{T} A_{22}^{*} P_{2} .
\end{aligned}
$$

Note

$$
\begin{aligned}
& \widehat{B}=U\left(\begin{array}{cc}
\Sigma_{1} V_{1}^{T} \Lambda V_{1} \Sigma_{1}^{-1} & U_{1}^{T} A_{11}^{*} U_{2} \\
0 & {\left[B_{22}\right]_{D_{r}}}
\end{array}\right) U^{T}, \\
& \widehat{C}=P\left(\begin{array}{cc}
\Sigma_{2} Q_{1}^{T} \Lambda Q_{1} \Sigma_{2}^{-1} & P_{1}^{T} A_{22}^{*} P_{2} \\
0 & {\left[C_{22}\right]_{D_{r}}}
\end{array}\right) P^{T} .
\end{aligned}
$$

Equations (55), (56), and (25) imply equation (42).

\section{Numerical Example}

Based on Theorems 1 and 2, we propose the following algorithm for solving Constrained Inverse Eigenproblem and Optimal Approximation Problem (Algorithm 1).

In this section, we will give a numerical example to illustrate our results. All the tests are performed by MATLAB6.5.

The matrices $X, \Lambda$, and $A^{*}$ and the radius $r$ are given by following: 


$$
\begin{aligned}
& X=\left(\begin{array}{ccc}
-0.0638 & -0.1385 & -0.0249 \\
-0.0593 & -0.3027 & -0.0061 \\
-0.3301 & 0.0521 & 0.0347 \\
0.6192 & 0.6202 & 0.0000 \\
0.6192 & -0.6202 & 0.0000 \\
-0.3301 & -0.0521 & -0.0347 \\
-0.0593 & 0.3027 & 0.0061 \\
-0.0638 & 0.1385 & 0.0249
\end{array}\right) \\
& \Lambda=\left(\begin{array}{ccc}
0.7611 & 0.0000 & 0.0000 \\
0.0000 & 0.5529 & 0.0702 \\
0.0000 & -0.0702 & 0.5529
\end{array}\right) \text {, } \\
& A^{*}=\left(\begin{array}{llllllll}
0.9501 & 0.8214 & 0.9355 & 0.1389 & 0.4451 & 0.8381 & 0.3046 & 0.3784 \\
0.2311 & 0.4447 & 0.9169 & 0.2028 & 0.9318 & 0.0196 & 0.1897 & 0.8600 \\
0.6068 & 0.6154 & 0.4103 & 0.1987 & 0.4660 & 0.6813 & 0.1934 & 0.8537 \\
0.4860 & 0.7919 & 0.8936 & 0.6038 & 0.4186 & 0.3795 & 0.6822 & 0.5936 \\
0.8913 & 0.9218 & 0.0579 & 0.2722 & 0.8462 & 0.8318 & 0.3028 & 0.4966 \\
0.7621 & 0.7382 & 0.3529 & 0.1988 & 0.5252 & 0.5028 & 0.5417 & 0.8998 \\
0.4565 & 0.1763 & 0.8132 & 0.0153 & 0.2026 & 0.7095 & 0.1509 & 0.8216 \\
0.0185 & 0.4057 & 0.0099 & 0.7468 & 0.6721 & 0.4289 & 0.6979 & 0.6449
\end{array}\right), \\
& r=0.4 \text {. }
\end{aligned}
$$

By algorithm, the constrained optimal approximation solution $\widehat{A}$ by Optimal Approximation Problem is

$$
\widehat{A}=\left(\begin{array}{cccccccc}
0.4539 & 0.2884 & -0.1728 & 0.0469 & -0.1080 & 0.0782 & 0.0071 & -0.0710 \\
0.2569 & -0.0060 & -0.1473 & -0.0179 & -0.0476 & 0.2079 & -0.3727 & -0.1464 \\
0.1450 & 0.1242 & -0.0729 & -0.2928 & -0.2391 & -0.3526 & 0.0205 & 0.6977 \\
0.0832 & 0.2522 & 0.3522 & 0.5442 & 0.2411 & -0.5450 & 0.3983 & 0.5450 \\
0.5450 & 0.3983 & -0.5450 & 0.2411 & 0.5442 & 0.3522 & 0.2522 & 0.0832 \\
0.6977 & 0.0205 & -0.3526 & -0.2391 & -0.2928 & -0.0729 & 0.1242 & 0.1450 \\
-0.1464 & -0.3727 & 0.2079 & -0.0476 & -0.0179 & -0.1473 & -0.0060 & 0.2569 \\
-0.0710 & 0.0071 & 0.0782 & -0.1080 & 0.0469 & -0.1728 & 0.2884 & 0.4539
\end{array}\right) .
$$

In the practical engineering problems, it is usually required that the matrix is a centrosymmetric matrix and the partial eigenvalues of the matrix must be located in a given closed disc or interval. We verify that $\widehat{A}$ in Optimal Approximation Problem is a centrosymmetric matrix and the eigenvalues of $\lambda(\widehat{A}) / \lambda(\Lambda)$ are lost in the disc $D_{r}$.

\section{Data Availability}

All data generated or analyzed during this study are included in this article.

\section{Conflicts of Interest}

The authors declare that they have no conflicts of interest.

\section{Acknowledgments}

Research supported by the Scientific Research of Hunan Provincial Education Department (Grant no. 18B419) and Science and Technology Project of Changsha (Grant no. kc1809026). 


\section{References}

[1] L. R. Fletcher, "An inverse eigenvalue problem from control theory," in Numerical Treatment of Inverse Problems for Differential and Integral Equations, P. Deuflhard, E. Hairer, and B. Birkhauser, Eds., pp. 161-170, Springer, Berlin, Germany, 1983.

[2] W. M. Wonham, Linear Multivariable Control. A Geometric Approach, Springer-Verlag, Berlin, Germany, 1979.

[3] V. Barcilon, "Inverse problem for a vibration beam," Journal of Applied Mathematics and Physics ZAMP, vol. 27, pp. 346-358, 1976.

[4] G. M. L. Gladwell, Inverse Problems in Vibration, Martinus Nijhoff, Dordrecht, The Netherlands, 1986.

[5] K. T. Jeseph, "Inverse eigenvalue problem in structural design," AIAA Journal, vol. 30, no. 12, pp. 2890-2896, 1992.

[6] S. Friedland, "The reconstruction of a symmetric matrix from the spectral data," Journal of Mathematical Analysis and Applications, vol. 71, no. 2, pp. 412-422, 1979.

[7] L. Aceto and D. Trigiante, "The stability problem for linear multistep methods: old and new results," Journal of Computational and Applied Mathematics, vol. 210, no. 1-2, pp. 2-12, 2007.

[8] L. Aceto and D. Trigiante, "On the A-stable methods in the GBDF class," Nonlinear Analysis: Real World Applications, vol. 3, no. 1, pp. 9-23, 2002.

[9] Z. Y. Peng, X. Y. Hu, and L. Zhang, "The inverse problem of centrosymmetric matrices with a submatrix constraint," Journal of Computational Mathematics, vol. 22, no. 4, pp. 535-544, 2004.

[10] F. Z. Zhou, X. Y. Hu, and L. Zhang, "The optimal approximation to centro-symmetric matrices on the linear manifold," Applied Mathematics-A Journal of Chinese Universities, vol. 3, pp. 265-272, 2002, in Chinese.

[11] F. Z. Zhou, Z. Z. Zhang, and X. Y. Hu, "The inverse eigenvalue problem for centro-symmetric nonnegative definite matrices," Journal of Hunan Educational Institute, vol. 29, no. 1, pp. 24-28, 2002, in Chinese.

[12] F.-Z. Zhou, X.-Y. Hu, and L. Zhang, "The solvability conditions for the inverse eigenvalue problems of centro-symmetric matrices," Linear Algebra and Its Applications, vol. 364, pp. 147-160, 2003.

[13] S. Somphotphisut and K. Wiboonton, "On the normal centrosymmetric nonnegative inverse eigenvalue problem," 2017.

[14] M. zhang and D. Y. Liu, "Least-square solution s of inverse problems for symmetric and generalized centrosymmetric matrices," Journal of Hubei University for Nationalities, Natural Science Edition, vol. 29, pp. 241-244, 2011, in chinese.

[15] S. Q. Zhou and H. Dai, The Algebraic Inverse Eigenvalue Problem, Henan Science and Technology Press, Zhengzhou, China, 1991.

[16] M. T. Chu and G. H. Golub, Inverse Eigenvalue Problems, Oxford Science Publications, Oxford, NY, USA, 2005.

[17] S. Gigola, L. Lebtahi, and N. Thome, "The inverse eigenvalue problem for a hermitian reflexive matrix and the optimization problem," Journal of Computational and Applied Mathematics, vol. 291, pp. 449-457, 2016.

[18] L. Zhang, "A class of inverse eigenvalue problems of symmetric matrices," Numerical Mathematics: A Journal of Chinese Universities, vol. 12, no. 1, pp. 65-71, 1991.

[19] G.-X. Huang and F. Yin, "Constrained inverse eigenproblem and associated approximation problem for anti-hermitian R-symmetric matrices," Applied Mathematics and Computation, vol. 186, no. 1, pp. 426-434, 2007.
[20] X.-Y. Peng, H.-J. Xiong, and W. Liu, “The constrained inverse eigenvalue problem and its approximation for normal matrices," Linear Algebra and Its Applications, vol. 435, no. 12, pp. 3115-3123, 2011.

[21] X.-P. Pan, X.-Y. Hu, and L. Zhang, "A class of constrained inverse eigenproblem and associated approximation problem for skew symmetric and centrosymmetric matrices," Linear Algebra and Its Applications, vol. 408, no. 1, pp. 66-77, 2005.

[22] X.-P. Pan, X.-Y. Hu, and L. Zhang, "The solvability conditions for a class of constrained inverse eigenvalue problem of antisymmetric matrices," Journal of the Korean Mathematical Society, vol. 43, no. 1, pp. 87-98, 2006.

[23] J. L. Zhen and X. H. Zhen, Special Matrices, Qinghua University Press, Beijing, China, 2001, in Chinese.

[24] R. A. Hong and C. R. Johnson, Matrix Analysis, Cambridge University Press, New York, NY, USA, 1990. 